CEP 17-02

\title{
Do Merger Efficiencies Always Mitigate Price Increases?
}

Zhiqi Chen

Carleton University
Gang Li

Nanjing University

January 2017

\section{CARLETON ECONOMIC PAPERS}
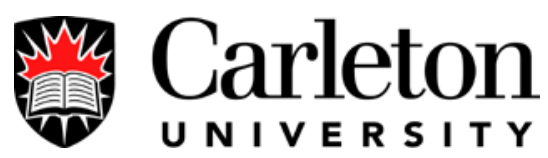

Department of Economics

1125 Colonel By Drive Ottawa, Ontario, Canada K1S $5 B 6$ 


\title{
Do Merger Efficiencies Always Mitigate Price Increases? ${ }^{\dagger}$
}

\author{
by ZHIQI CHEN* AND GANG LI **
}

December 12, 2016

\begin{abstract}
In a Cournot model with differentiated products, we demonstrate that merger efficiencies in the form of lower marginal costs for the merging firms (the insiders) lead to higher postmerger prices under certain conditions. Specifically, when the degree of substitutability is low between the products offered by the two insiders but high between those by an insider and an outsider, increased merger efficiencies may exert upward rather than downward pressure on the prices of the merging firms. Our results suggest that in cases where firms engage in quantity competition, antitrust authorities should not presume that merger efficiencies will necessarily mitigate the anticompetitive effects of the merger. Prices can go up because of large efficiencies.
\end{abstract}

Key words: Merger efficiencies, Cournot model, Product differentiation

JEL Classification: L13, L40

*Department of Economics, Carleton University, Canada; email: zhiqi.chen@carleton.ca.

** School of Economics, Nanjing University, China; email: gangli@nju.edu.cn.

$\dagger$ We would like to thank Yongmin Chen, Gamal Atallah, Justin Johnson, Ping Lin, Chengzhong Qin, Aggey Semenov, Adam Wong, Wen Zhou, three anonymous referees and seminar participants at University of Manitoba and Shanghai University of Finance and Economics for their comments. 


\section{Introduction}

Mergers can create efficiencies, arising from sources such as scale economy, rationalized production schedule between plants, and acquisition of complementary technologies. It is widely accepted by economists that lower marginal costs brought about by merger efficiencies will encourage firms to compete more aggressively, thus mitigating the loss of competition that may be caused by a merger. As Werden (1996, p.409) has noted, "If a merger caused a reduction in marginal cost for the merging firms, the cost reduction would offset the anticompetitive effect of the merger on prices. Indeed, if the merger reduced the marginal costs of the merging firms by a sufficient amount, it would cause all prices in the industry to fall."

Consistent with this conventional wisdom among economists, antitrust authorities typically associate the amount of price reduction with the magnitude of merger efficiencies. For example, the US Horizontal Merger Guidelines (2010, pp.30-31) states, "the Agencies consider whether cognizable efficiencies likely would be sufficient to reverse the merger's potential to harm customers in the relevant market, e.g., by preventing price increases in that market." The EC Guidelines on the Assessment of Horizontal Mergers (2004, paragraph 79) indicates that "the relevant benchmark in assessing efficiency claims is that consumers will not be worse off as a result of the merger. For that purpose, efficiencies should be substantial..." In Canada, the Competition Act contains a provision that instructs the Competition Tribunal not to block a merger that "has brought about or is likely to bring about gains in efficiency that will be greater than, and will offset, the effects of any prevention or lessening of competition that will result or is likely to result from the merger..." Indeed, in discussions of merger enforcement policies and practices it is often held as self-evident that merger efficiencies mitigate anticompetitive effects and exert downward pricing pressure (see, e.g., Fisher et al. 1989 and Salop 1987). 
In this paper, we challenge the conventional wisdom that merger efficiencies in the form of lower marginal costs will always counteract the price increases arising from the loss of competition. We do so by examining the effects of a merger in a model where firms produce differentiated products and compete in quantities. The merger generates efficiencies so that the marginal costs of the merging firms (the insiders) are lower after the merger. We show that an increase in merger efficiencies may either raise or reduce the post-merger prices of the insiders, depending on the degrees of substitutability among the products offered by the insiders and their rivals (the outsiders) as well as the number of competitors. Specifically, increased merger efficiencies exert upward - rather than downward - pressure on the prices if the degree of substitutability is low between the two insiders but high between the insiders and outsiders, and the number of competitors is not too small.

In the literature, theoretical analyses of merger efficiencies are typically conducted in the framework of Cournot oligopoly. ${ }^{1}$ They include Farrell and Shapiro (1990), Levin (1990), Cheung (1992), Froeb and Werden (1998), Motta and Vasconcelos (2005), Banal-Estañol et al. (2008), Amir et al. (2009), Jovanovic and Wey (2012). All of them assume that firms produce a homogenous product, in which case a reduction in marginal costs after a merger always leads to a lower price. ${ }^{2}$ In contrast, our analysis shows that the effects of lower marginal costs depend critically on the degrees of product differentiation.

This paper is organized as follows. The model is presented in section 2, and the price effects of merger efficiencies are analyzed in section 3. Sections 4 and 5 examine the overall effects of

\footnotetext{
${ }^{1}$ One exception is Werden (1996), which studies merger efficiencies in a differentiated Bertrand model.

${ }^{2}$ In particular, Froeb and Werden (1998) derive a formula for the amount of reduction in marginal costs needed to prevent a merger from increasing price in a Cournot industry.
} 
the merger on profits and prices, while section 6 considers the impact of merger efficiencies on consumer welfare. Section 7 concludes.

\section{The Model}

Consider an industry where firms produce differentiated goods and compete in quantities.

Initially, there are $n(\geq 3)$ firms. Each firm produces one good at constant marginal cost, denoted by $c$. Then two of these firms, firm 1 and firm 2, decide to merge.

We are interested in a situation where the merger is motivated primarily by efficiency gains. ${ }^{3}$ Specifically, suppose the merger generates efficiency gains that reduce the marginal costs of the merging firms to $(1-e) c$, where $e \in(0,1)$ measures the magnitude of merger efficiencies. The marginal costs of the other firms, on the other hand, are not affected by the merger. The focus of our analysis will be on how the price effects of the merger are influenced by the size of $e^{4}$

On the consumer side, we suppose that the demand functions are symmetric for the insiders and, respectively, for the outsiders. This enables us to analyze the equilibrium behavior of the insiders and outsiders as two groups. Specifically, suppose that firm $i(=1,2, \ldots, n)$ faces the following inverse demand function: $p_{i}=P^{i}\left(\mathbf{q}_{I}, \mathbf{q}_{o}\right)$, where $p_{i}$ is the price of product $i, \mathbf{q}_{I} \equiv$ $\left(q_{1}, q_{2}\right)$ the vector of quantities produced by the insiders (i.e., firm 1 and firm 2), and $\mathbf{q}_{o} \equiv$ $\left(q_{3}, \ldots, q_{n}\right)$ the vector of quantities produced by the outsiders (i.e., firms 3 through $n$ ). We will

\footnotetext{
${ }^{3}$ Geiger and Schiereck (2014) classifies the motives for mergers into three categories: the efficiency theory (which stipulates that mergers are motivated by efficiencies), the monopolistic collusion theory (which posits that mergers are executed to improve market positioning and to gain market power), and the agency and hubris theories (which associate mergers with agency problems or hubris of management). Empirical evidence for mergers motivated by efficiencies can be found in Lichtenberg and Siegel (1992), McGuckin and Nguyen (1995), Ollinger and Nguyen (2003), and Geiger and Schiereck (2014)

${ }^{4}$ In this analysis, we do not consider merger efficiencies in the form of a reduction in fixed costs because its implication for post-merger prices is obvious. Provided that the number of active outsiders remains the same after the merger, the reduction in the insiders' fixed costs will have no impact on post-merger prices of all firms.
} 
use subscript $j$ to denote the partial derivative of $P^{i}\left(\mathbf{q}_{I}, \mathbf{q}_{o}\right)$ with respective to $q_{j}$. Thus, $P_{j}^{i}$ (三 $\partial P^{i} / \partial q_{j}$ ) indicates the responsiveness of price $i$ to a small change in the quantity of good $j$. For $i \neq j, P_{j}^{i}$ measures the degree of substitutability between good $i$ and $\operatorname{good} j$.

The demand structure is symmetric in the sense that firms within each group have the same degree of substitutability when they produce the same quantity. Moreover, we assume each inverse demand function is separable in its arguments. These assumptions imply that the system of demand functions can be written in the following form:

$$
\begin{gathered}
P^{1}\left(\mathbf{q}_{I}, \mathbf{q}_{o}\right)=\Psi_{I}\left(q_{1}\right)+\Phi_{I}\left(q_{2}\right)+\sum_{j=3}^{n} \Phi_{O}\left(q_{j}\right), \\
P^{2}\left(\mathbf{q}_{I}, \mathbf{q}_{o}\right)=\Psi_{I}\left(q_{2}\right)+\Phi_{I}\left(q_{1}\right)+\sum_{j=3}^{n} \Phi_{O}\left(q_{j}\right), \\
P^{i}\left(\mathbf{q}_{I}, \mathbf{q}_{o}\right)=\Psi_{O}\left(q_{i}\right)+\sum_{j \neq i} \Phi_{O}\left(q_{j}\right), \quad i=3,4, \ldots n
\end{gathered}
$$

where $\Psi_{I}\left(q_{i}\right), \Psi_{O}\left(q_{i}\right), \Phi_{I}\left(q_{i}\right)$ and $\Phi_{O}\left(q_{i}\right)$ are twice continuously differentiable functions of $q_{i}$. Furthermore, we assume that these functions satisfy the following assumptions.

Assumptions 1. The goods are imperfect substitutes, that is, $\Psi_{I}^{\prime}(q)<\Phi_{I}^{\prime}(q)<0, \Psi_{I}^{\prime}(q)<$

$$
\Phi_{O}^{\prime}(q)<0 \text { and } \Psi_{O}^{\prime}(q)<\Phi_{O}^{\prime}(q)<0 .
$$

Assumption 2. The marginal revenue of each firm is a decreasing function of its own quantity both before and after merger, that is, $2 \Psi_{I}^{\prime}(q)+q \Psi_{I}^{\prime \prime}(q)<0, \quad 2 \Psi_{I}^{\prime}(q)+q \Psi_{I}^{\prime \prime}(q)+$ $q \Phi_{I}^{\prime \prime}(q)<0$ and $2 \Psi_{O}^{\prime}(q)+q \Psi_{O}^{\prime \prime}(q)<0 .^{5}$

To understand Assumption 1, observe that $P_{i}^{i}=\Psi_{I}^{\prime}$ and $P_{j}^{i}=\Phi_{I}^{\prime}$ for the insiders (i.e., $i, j=$ $1,2, i \neq j)$, and $P_{i}^{i}=\Psi_{O}^{\prime}$ and $P_{j}^{i}=\Phi_{O}^{\prime}$ for the outsiders (i.e., $i, j=3, \ldots n, i \neq j$ ). Therefore, $\Phi_{I}^{\prime}$

\footnotetext{
${ }^{5}$ Note that the marginal revenue of the merged entity with respect to $q_{i}(i=1,2)$ is $\Psi_{I}\left(q_{i}\right)+\Phi_{I}\left(q_{j}\right)+$ $\sum_{k=3}^{n} \Phi_{O}\left(q_{k}\right)+q_{i} \Psi_{I}^{\prime}\left(q_{i}\right)+q_{j} \Phi_{I}^{\prime}\left(q_{i}\right)$, where $j=1,2$ and $j \neq i$.
} 
(respectively, $\Phi_{O}^{\prime}$ ) measures the degree of substitutability between the insiders (respectively, any pair of outsiders). The degree of substitutability between an insider and an outsider is represented by $\Phi_{O}^{\prime}$ as well.

Since $\Phi_{I}(q)$ and $\Phi_{O}(q)$ can be different functions, this demand system admits the possibility that the degree of substitutability between the two insiders is different from that between an insider and an outsider. This, in turn, enables us to investigate how the effects of merger efficiencies are influenced by the degree of substitutability between the two insiders relative to that between an insider and an outsider. ${ }^{6}$

Given the symmetry in the demand system, we will focus our analysis on symmetric equilibriums in which the two insiders produce the same quantity and all outsiders choose the same output. We will continue to use subscript $I$ to denote the variables of an insider and subscript $O$ those of an outsider.

Before the merger, each firm solves the following profit-maximization problem:

$$
\max _{q_{i}}\left[P^{i}\left(\mathbf{q}_{I}, \mathbf{q}_{o}\right)-c\right] q_{i} .
$$

This yields the standard first-order condition:

$$
P^{i}+q_{i} P_{i}^{i}=c .
$$

We use superscript $C$ to indicate the pre-merger equilibrium. Accordingly, $q_{I}^{C}$ and $q_{O}^{C}$ denote the pre-merger quantity of an insider and an outsider, respectively. Similarly, $\pi_{I}^{C}$ and $\pi_{O}^{C}$ denote the pre-merger equilibrium profit of an insider and an outsider, respectively.

After the merger, the insiders' profit-maximization problem becomes

$$
\max _{q_{1}, q_{2}}\left[P^{1}\left(\mathbf{q}_{I}, \mathbf{q}_{o}\right)-(1-e) c\right] q_{1}+\left[P^{2}\left(\mathbf{q}_{I}, \mathbf{q}_{o}\right)-(1-e) c\right] q_{2} .
$$

\footnotetext{
${ }^{6}$ In practice, one factor considered by antitrust authorities in their assessment of the unilateral effects of a merger is whether the products of the merging parties are close substitutes relative to those offered by other competitors. See, for example, section 6.1 of the U.S. Horizontal Merger Guidelines (2010).
} 
Note in (6) that the post-merger marginal costs of the insiders are $(1-e) c$. The first-order conditions can be written in the form:

$$
P^{i}+q_{i} P_{i}^{i}+q_{j} P_{i}^{j}=(1-e) c \quad(i, j=1,2, i \neq j) .
$$

The optimization problem and the first-order condition of an outsider are the same as (4) and (5). ${ }^{7}$ The post-merger equilibrium quantities are then determined by (5) and (7).

Let $q_{I}^{M}$ and $q_{O}^{M}$ denote the post-merger quantity of an insider and an outsider, respectively. Then (5) and (7), along with (1) - (3), imply that $q_{I}^{M}$ and $q_{O}^{M}$ are determined by the following two equations:

$$
\begin{gathered}
\Psi_{I}\left(q_{I}^{M}\right)+\Phi_{I}\left(q_{I}^{M}\right)+(n-2) \Phi_{O}\left(q_{O}^{M}\right)+q_{I}^{M} \Psi_{I}^{\prime}\left(q_{I}^{M}\right)+q_{I}^{M} \Phi_{I}^{\prime}\left(q_{I}^{M}\right)=(1-e) c \\
\Psi_{O}\left(q_{O}^{M}\right)+2 \Phi_{O}\left(q_{I}^{M}\right)+(n-3) \Phi_{O}\left(q_{O}^{M}\right)+q_{o}^{M} \Psi_{O}^{\prime}\left(q_{O}^{M}\right)=c
\end{gathered}
$$

We use $\pi_{I}^{M}$ and $\pi_{O}^{M}$ to denote the post-merger equilibrium profit of an insider and an outsider, respectively.

\section{Price Effects of Merger Efficiencies}

The objective of this paper is to challenge the conventional wisdom that merger efficiencies in the form of lower marginal costs will always mitigate the upward pricing pressure arising from a horizontal merger. Hence, we start by examining the impact of merger efficiencies on the prices of the insiders and outsiders. Then we will move on to analyze the overall effects of the merger on profits and prices in sections 4 and 5.

We first consider the impact of merger efficiencies on equilibrium quantities. Conducting comparative statics on (8) and (9), we obtain:

$$
\frac{\partial q_{I}^{M}}{\partial e}=-\frac{c\left[2 \Psi_{O}^{\prime}+q_{o}^{M} \Psi_{O}^{\prime \prime}+(n-3) \Phi_{O}^{\prime}\left(q_{O}^{M}\right)\right]}{J}
$$

${ }^{7}$ Assumption 2 ensures that the second-order conditions of the firms' optimization problems are satisfied both before and after the merger. 


$$
\frac{\partial q_{O}^{M}}{\partial e}=\frac{2 c \Phi_{O}^{\prime}\left(q_{I}^{M}\right)}{J}
$$

Where $J \equiv\left[2\left(\Psi_{I}^{\prime}+\Phi_{I}^{\prime}\right)+q_{I}^{M}\left(\Psi_{I}^{\prime \prime}+\Phi_{I}^{\prime \prime}\right)\right]\left[2 \Psi_{O}^{\prime}+(n-3) \Phi_{O}^{\prime}\left(q_{O}^{M}\right)+q_{o}^{M} \Psi_{O}^{\prime \prime}\right]-2(n-2)$

$\Phi_{O}^{\prime}\left(q_{I}^{M}\right) \Phi_{O}^{\prime}\left(q_{O}^{M}\right)$ is the Jacobian matrix associated with (8)-(9). For simplicity, we have omitted the arguments in the functions $\Psi_{I}\left(q_{I}\right), \Phi_{I}\left(q_{I}\right)$ and $\Psi_{O}\left(q_{O}\right)$. But we have retained the argument in the function $\Phi_{O}\left(q_{i}\right)$ to avoid ambiguity.

From (10) and (11), we observe:

Lemma 1. $\partial q_{I}^{M} / \partial e>0$ and $\partial q_{O}^{M} / \partial e<0$ if and only if $J>0$.

Proof. The numerators of (10) and (11) are negative because $2 \Psi_{O}^{\prime}+q_{O} \Psi_{O}^{\prime \prime}<0$ and $\Phi_{O}^{\prime}<0$. Hence, the signs of (10) and (11) are determined by the sign of $J$, as described in Lemma 1.

Lemma 1 states that larger merger efficiencies increase the quantity of each insider but reduce the quantity of each outsider as long as $J>0$. If $J<0$, on the other hand, we would have a perverse situation where a reduction in the marginal cost of the merged entity causes insiders to contract output and outsiders to expand output. To rule out this perverse situation, we assume: ${ }^{8}$

Assumption 3. $J>0$.

We are now in a position to present the core result of this paper.

Proposition 1. A larger $e$ leads to higher prices for the insiders if and only if

$$
\frac{\Phi_{I}^{\prime}\left(q_{I}^{M}\right)}{\Psi_{I}^{\prime}\left(q_{I}^{M}\right)}<\frac{\Phi_{O}^{\prime}\left(q_{I}^{M}\right)}{\Psi_{I}^{\prime}\left(q_{I}^{M}\right)} \cdot \frac{2(n-2) \Phi_{O}^{\prime}\left(q_{O}^{M}\right)}{\left[2 \Psi_{O}^{\prime}+q_{O}^{M} \Psi_{O}^{\prime \prime}+(n-3) \Phi_{O}^{\prime}\left(q_{O}^{M}\right)\right]}-1
$$

holds in the post-merger equilibrium.

Proof. Using (1), (10) and (11), we can show that

${ }^{8}$ Recall that the main objective of this paper is to demonstrate that merger efficiencies can lead to higher prices. This result would not be very convincing if it were obtained from a perverse situation where a lower marginal cost reduces the insiders' quantities and increases the outsiders' quantities. 


$$
\frac{\partial p_{I}^{M}}{\partial e}=\frac{c\left[2(n-2) \Phi_{O}^{\prime}\left(q_{I}^{M}\right) \Phi_{O}^{\prime}\left(q_{O}^{M}\right)-\left(\Psi_{I}^{\prime}+\Phi_{I}^{\prime}\right)\left(2 \Psi_{O}^{\prime}+q_{o}^{M} \Psi_{O}^{\prime \prime}+(n-3) \Phi_{O}^{\prime}\left(q_{O}^{M}\right)\right)\right]}{J}
$$

Since $J>0$, the sign of (13) is determined by the sign of its numerator. The latter is positive if and only if (12) holds.

Proposition 1 suggests that larger efficiencies can indeed lead to higher post-merger prices for the insiders under some circumstances. A close examination of (12) indicates that this would arise if the degree of substitutability between the products of the two insiders, as measured by $\Phi_{I}^{\prime}\left(q_{I}^{M}\right) / \Psi_{I}^{\prime}\left(q_{I}^{M}\right)$, is relatively low and that between the products of an insider and an outsider, $\Phi_{O}^{\prime}\left(q_{I}^{M}\right) / \Psi_{I}^{\prime}\left(q_{I}^{M}\right)$, is relatively high.

Condition (12) is key to the understanding of Proposition 1. To flush out the implications of this condition, we consider the special case of a linear demand system. Specifically, suppose the inverse demand functions take the following form:

$$
\begin{aligned}
& p_{1}=\alpha-q_{1}-\gamma q_{2}-\delta \sum_{j=3}^{n} q_{j} \\
& p_{2}=\alpha-\gamma q_{1}-q_{2}-\delta \sum_{j=3}^{n} q_{j} \\
& p_{i}=\alpha-q_{i}-\delta \sum_{j \neq i} q_{j}(i=3,4, \ldots, n),
\end{aligned}
$$

In (14)-(16), $\alpha>c$ and the values of $\gamma$ and $\delta$ are strictly between 0 and 1.

Relating to the general demand functions in (1)-(3), we now have $\Psi_{I}^{\prime}=\Psi_{O}^{\prime}=-1, \Phi_{I}^{\prime}=-\gamma$, and $\Phi_{O}^{\prime}=-\delta$. Accordingly, $\gamma$ measures the degree of substitutability between the two insiders (firms 1 and 2), while $\delta$ indicates the degree of substitutability between an insider and an outsider as well as that between any pair of outsiders. A larger $\gamma$ (respectively, $\delta$ ) means that the goods produced by the two insiders (respectively, by an insider and an outsider) are closer 
substitutes. In addition, it is easy to verify that this system of linear demand functions satisfy Assumptions 1-3.

Using the linear demand functions, we can obtain the closed-form solutions to the equilibrium quantities and prices before and after the merger. We present these and other details of this special case in Appendix A.

Regarding our key condition (12), we use the linear demand functions (14)-(16) to find the following conditions.

Proposition 2. Suppose the market demand functions are linear, as represented by (14)-(16). A larger $e$ leads to higher prices for the insiders if and only if

$$
\gamma<\frac{2(n-2) \delta^{2}}{(n-3) \delta+2}-1
$$

To satisfy (17), it is necessary that $\delta>1 / 2$ and

$$
n>\frac{2+\delta(4 \delta-3)}{\delta(2 \delta-1)}
$$

Proof. Condition (17) is obtained by rewriting (12) using the linear demand system (14)-(16). Since $\gamma>0$, the right-hand side of (17) has to be positive. To satisfy the latter, we need $2(n-2) \delta^{2}>(n-3) \delta+2$. Rearranging terms, we find

$$
\delta(2 \delta-1) n>2+\delta(4 \delta-3)
$$

It can be shown that the right-hand side of (19) is strictly positive. Hence, to satisfy (19), it is necessary that $\delta>1 / 2$ (to ensure that the left-hand side is positive). Solving (19) for $n$, we obtain (18).

Proposition 2 provides more clarity to the finding in Proposition 1. It clearly shows that merger efficiencies exert upward pressure on the prices of the merging firms if the degree of substitutability is low between the two products of the insiders but high between those of an 
insider and an outsider. For this to occur, moreover, the number of competitors cannot be too small.

To understand the intuition behind Propositions 1 and 2, rewrite the insiders' demand function (14) as:

$$
p_{I}=\alpha-(1+\gamma) q_{I}-\delta(n-2) q_{O} .
$$

From (20), we can see that merger efficiencies affect the insiders' prices through two channels. The first channel is through each insider's output $q_{I}$. Lemma 1 states that lower marginal costs induce the insiders to expand output after the merger. This is the direct effect of merger efficiencies on the post-merger prices of the insiders. The second channel is through every outsider's output $q_{o}$. In Lemma 1, we see that larger merger efficiencies cause each outsider to reduce its output. This is the strategic effect arising from the fact that outputs are strategic substitutes. As the insiders expand outputs in response to their lower marginal costs, the outsiders react by contracting their outputs. The latter tends to push up the insiders' prices.

As we can see from (20), the magnitude of the direct effect decreases with the degree of substitutability between the two insiders $(\gamma)$, while the aggregate of the strategic effect for all outsiders increases with the number of competitors $(n)$ and the degree of substitutability between the products of an insider and an outsider $(\delta)$. Accordingly, the strategic effect dominates the direct effect if $n$ and $\delta$ are sufficiently large and $\gamma$ is sufficiently small.

Three points about the conditions in Proposition 2 are worth noting. First, condition (17) implies that $\gamma<\delta$, i.e., the degree of substitutability between the insiders is smaller than that between an insider and an outsider. In other words, larger merger efficiencies lead to higher prices only if the insiders' products are more distant substitutes for each other than for those of 
the outsiders. Note that this is the type of situations where a merger would normally be considered as unlikely to cause price increases. ${ }^{9}$

To expand on this point, consider the extreme case where the demands for the products of the two insiders are independent of each other, i.e., $\gamma=0 .{ }^{10}$ In this case, the merger would not have any effect on quantities and prices in the absence of efficiency gains. By the conventional wisdom, such a merger should not raise any competition concerns because the only motivation for this merger is to reap efficiency gains. Yet in this model, such a merger will actually cause the prices of the merging firms to rise if $\delta>1 / 2$ and $n$ satisfies (18). Here, the merger leads to higher prices because of, rather than despite of, merger efficiencies.

Second, the positive relationship between post-merger prices and efficiencies does not arise if the firms produce a homogeneous good. To be more precise, the conditions in Proposition 2 cannot be satisfied by $\gamma=\delta=1$. This is why merger efficiencies always lead to a lower price in the existing merger literature based on homogenous Cournot models (e.g., Farrell and Shapiro 1990).

Third, condition (18) does not necessarily mean that this industry is unconcentrated. It can be shown that the expression on the right-hand side of $(18)$ is decreasing in $\delta \in(1 / 2,1]$ and is equal to 3 at $\delta=1$. In other words, (18) could be satisfied in an industry with only four firms before the merger.

Next, we consider the effects of merger efficiencies on the outsiders' prices. Going back to the general model, we use the comparative statics results (8) and (9) to derive the following result.

\footnotetext{
9 "A merger is unlikely to generate substantial unilateral price increases if non-merging parties offer very close substitutes for the products offered by the merging firms" (US Horizontal Merger Guidelines 2010 section 6.1).

${ }^{10}$ We thank an anonymous referee for the suggestion that we consider this special case.
} 
Proposition 3. A larger $e$ leads to higher prices for each outsider if and only if

$$
\Psi_{O}^{\prime}+q_{O}^{M} \Psi_{O}^{\prime \prime}>0
$$

holds in the post-merger equilibrium.

Proof. Using (3), (8) and (9), we find:

$$
\frac{\partial p_{O}^{M}}{\partial e}=-\frac{2\left(\Psi_{O}^{\prime}+q_{o}^{M} \Psi_{O}^{\prime \prime}\right) c \Phi_{O}^{\prime}\left(q_{I}^{M}\right)}{J}
$$

Since $J>0$ and $\Phi_{O}^{\prime}<0,(22)$ is positive if and only if $\Psi_{O}^{\prime}+q_{O}^{M} \Psi_{O}^{\prime \prime}>0$.

Note that a necessary condition for (21) to hold is that $\Psi_{O}^{\prime \prime}>0$. If $\Psi_{O}^{\prime \prime} \leq 0$, on the other hand, a larger $e$ would lead to lower prices for outsiders. This implies that in the case of linear demand functions (where $\Psi_{O}^{\prime \prime}=0$ ), larger merger efficiencies lead to lower post-merger prices for the outsiders.

Intuitively, the effects of merger efficiencies on the outsiders' prices can go either way because of two opposing forces implied by Lemma 1. On the one hand, a larger $e$ reduces the post-merger quantity of each outsider, which tends to raise the price of an outsider. On the other hand, larger merger efficiencies cause the insiders to expand output, which tends to push down the price of an outsider. The net effect is, in general, ambiguous.

It is important to note that (21) in Proposition 3 and (12) in Proposition 1 are not mutually exclusive. In other words, it is possible that both conditions hold at the same time, and thus, larger merger efficiencies may raise the prices of both insiders and outsiders. In section 6, we will present a demand system under which this can indeed occur.

\section{Merger Profitability}

In this section, we investigate the effects of the merger on the profits of the insiders and outsiders. Since we have used a Cournot model of oligopoly, our analysis would not be complete without addressing the merger paradox. It is well-known in the literature that a merger is usually 
not profitable (for the merging firms) in a homogeneous Cournot model if the merger generates no efficiency gains. ${ }^{11}$ In the present model, however, the merger can generally be profitable because of product differentiation and merger efficiencies. Below we characterize the conditions under which the merger is indeed profitable.

Differentiating an insider's post-merger profit, we can easily confirm that $\partial \pi_{I}^{M} / \partial e>0$; that is, larger merger efficiencies improve the insiders' profitability. Therefore, it is reasonable to expect that the merger should be profitable for the insiders if merger efficiencies are sufficiently large.

To establish formally the above result in the general model, we need an additional assumption. Recall from Lemma 1 that the quantity of an insider rises, and the quantity of an outsider falls, with the size of efficiencies $e$. It is then possible that if $e$ is sufficiently large, the post-merger quantity of an insider could be larger than its pre-merger quantity. In this and the next section, we assume that this is indeed the case at $e=1$, that is,

Assumption 4. $q_{I}^{M}>q_{I}^{C}$ if $e=1$,

Intuitively, $e=1$ means that each insider's marginal cost falls to 0 after the merger. Therefore, if $c$ is sufficiently large to start with, a merger with $e=1$ would confer the insiders such a large cost advantage that they produce larger quantities than before the merger. Indeed, in the special case of linear demand functions, it is straightforward to show that Assumption 4 is satisfied if $c$ exceeds a certain threshold. ${ }^{12}$

Recall that $\pi_{I}^{C}$ and $\pi_{I}^{M}$ denote an insider's profit before and after the merger, respectively. Hence, the merger is profitable for the insiders if $\pi_{I}^{M}>\pi_{I}^{C}$.

\footnotetext{
${ }^{11}$ See, for example, Salant, et al. (1983) and Lommerud and Sørgard (1997).

12 See condition (A6) in Appendix A for details.
} 
Proposition 4. There exists an $e_{\pi} \in[0,1)$ such that $\pi_{I}^{M}>\pi_{I}^{C}$ if $e>e_{\pi}$.

Proof. Note that an outsider's best-response function is the same before and after the merger.

Accordingly, (9) represents a relationship between an outsider's quantity and an insider's

quantity both before and after the merger. We solve (9) to express $q_{O}$ as a function of $q_{I}$, denoted by $q_{O}=\tilde{q}_{O}\left(q_{I}\right)$. Then $q_{O}^{C}=\tilde{q}_{O}\left(q_{I}^{C}\right)$ and $q_{O}^{M}=\tilde{q}_{O}\left(q_{I}^{M}\right)$. Differentiating (9), we find:

$$
\tilde{q}_{O}^{\prime}\left(q_{I}\right)=-\frac{2 \Phi_{O}^{\prime}}{\Psi_{O}^{\prime}+q_{O} \Psi_{O}^{\prime \prime}+(n-3) \Phi_{O}^{\prime}}<0
$$

Assumption 4 and (23) imply that $q_{O}^{M}<q_{O}^{C}$ at $e=1$.

To compare $\pi_{I}^{M}$ with $\pi_{I}^{C}$ at $e=1$, note that $\pi_{I}^{M}=P^{1}\left(\mathbf{q}_{I}^{M}, \mathbf{q}_{O}^{M}\right) q_{I}^{M}>P^{1}\left(\mathbf{q}_{I}^{C}, \mathbf{q}_{O}^{M}\right) q_{I}^{C}$ because $\mathbf{q}_{I}^{M}$ is the insiders' best response to $\mathbf{q}_{O}^{M}$. Then $q_{O}^{M}<q_{O}^{C}$ implies $P^{1}\left(\mathbf{q}_{I}^{C}, \mathbf{q}_{O}^{M}\right) q_{I}^{C}>P^{1}\left(\mathbf{q}_{I}^{C}, \mathbf{q}_{O}^{C}\right) q_{I}^{C}$ $>\left[P^{1}\left(\mathbf{q}_{I}^{C}, \mathbf{q}_{O}^{C}\right)-c\right] q_{I}^{C}=\pi_{I}^{C}$. Hence, $\pi_{I}^{M}>\pi_{I}^{C}$ at $e=1$.

Differentiating $\pi_{I}^{M}$ and applying the envelope theorem, we find

$$
\frac{\partial \pi_{I}^{M}}{\partial e}=c q_{I}+(n-2) \Phi_{O}^{\prime}\left(q_{O}^{M}\right) \tilde{q}_{O}^{\prime} \frac{\partial q_{I}^{M}}{\partial e}>0
$$

If $\pi_{I}^{M}<\pi_{I}^{C}$ at $e=0$, there exists a unique $e_{\pi} \in(0,1)$ such that $\pi_{I}^{M}=\pi_{I}^{C}$ at $e=e_{\pi}$ and $\pi_{I}^{M}>$ $\pi_{I}^{C}$ for $e>e_{\pi}$. On the other hand, if $\pi_{I}^{M}>\pi_{I}^{C}$ at $e=0$, we set $e_{\pi}=0$.

Proposition 4 confirms the conjecture that the merger is profitable for the insiders when merger efficiencies are sufficiently large. Next, we move on to consider the effects of the merger on the outsiders' profits. Recall from Lemma 1 that larger merger efficiencies cause the outsiders to reduce output. Hence, we expect the post-merger profit of each outsider to fall below its pre-merger level if merger efficiencies are sufficiently large. This brings us to another critical value of $e$.

Lemma 2. There exists an $e_{p} \in\left(e_{\pi}, 1\right)$ such that $q_{I}^{M}=q_{I}^{C}, q_{O}^{M}=q_{O}^{C}, p_{I}^{M}=p_{I}^{C}$ and $p_{O}^{M}=p_{O}^{C}$ at $e=e_{p}$ 
Proof. Note that the left-hand side of (7) is less than that of (5) for $q_{i}=q_{j}=q_{I}$. This implies that if there is no merger efficiencies (i.e., if $e=0$ ), an insider's marginal revenue after the merger is lower than that before the merger. Since the best-response function of each outsider is the same before and after the merger, this implies that $q_{I}^{M}<q_{I}^{C}$ and $q_{O}^{M}>q_{O}^{C}$ at $e=0$. On the other hand, Assumption 4 and (23) imply that $q_{I}^{M}>q_{I}^{C}$ and $q_{O}^{M}<q_{O}^{C}$ at $e=1$. By the continuity of $q_{I}^{M}$ in $e$, there exists $e_{p} \in(0,1)$ such that $q_{I}^{M}=q_{I}^{C}$. Then $(23)$ implies $q_{O}^{M}=q_{O}^{C}$ at $e=e_{p}$. From the inverse demand functions (1)-(3), we infer that $p_{I}^{M}=p_{I}^{C}$ and $p_{O}^{M}=p_{O}^{C}$ at $e=e_{p}$.

In the case where $e_{\pi}=0, e_{p}>0$ implies that $e_{p}>e_{\pi}$. Now suppose $e_{\pi}>0$. Note that $\left[P^{i}\left(\mathbf{q}_{I}, \mathbf{q}_{o}\right)-(1-e) c\right] q_{i}>\left[P^{i}\left(\mathbf{q}_{I}, \mathbf{q}_{o}\right)-c\right] q_{i}$ for $e>0$. Hence, $\pi_{I}^{M}>\pi_{I}^{C}$ at $e=e_{p}$. Since $\pi_{I}^{M}=\pi_{I}^{C}$ at $e=e_{\pi},(24)$ implies that $e_{p}>e_{\pi}$.

Lemma 2 defines a critical value of $e$ such that the equilibriums before and after the merger involve exactly the same quantities and prices. Note that $e_{p}>e_{\pi}$, implying that the merger is profitable for the insiders even before their quantities reach their pre-merger levels. For the outsiders, on the other hand, $e_{p}$, represents a turning point for their post-merger profits vis-à-vis their pre-merger levels.

Proposition 5. $\pi_{O}^{M}<\pi_{O}^{C}$ if and only if $e>e_{p}$.

Proof. Since (4) represents an outsider's profit both before and after the merger, Lemma 2 implies that $\pi_{O}^{M}=\pi_{O}^{C}$ at $e=e_{p}$. Differentiating an outsider's post-merger profit with respect to $e$ and applying the envelope theorem, we obtain:

$$
\frac{\partial \pi_{O}^{M}}{\partial e}=2 q_{O}^{M} \Phi_{O}^{\prime}\left(q_{I}^{M}\right) \frac{\partial q_{I}^{M}}{\partial e}<0,
$$

in which $\partial q_{I}^{M} / \partial e>0$ by Lemma 1. Then (25) implies $\pi_{O}^{M}<\pi_{O}^{C}$ if and only if $e>e_{p}$. 
Proposition 5 indicates that the impact of the merger on the outsiders' profits depends on the size of merger efficiencies. The outsiders benefit from the merger if efficiencies are small but are harmed by it if efficiencies are large. This observation suggests that the expected impact of a merger on the outsiders' profits is not a good indicator of the presence or absence of merger efficiencies. $^{13}$

\section{Price Effects of Merger}

Recall from Proposition 1 that the prices of the insiders rise with the size of merger efficiencies under condition (12). This result suggests an intriguing possibility that a merger could lead to lower prices when efficiencies are small but higher prices when efficiencies are large. We investigate this possibility in this section.

Lemma 2 identifies a critical level of merger efficiencies, $e_{p}$, around which the merger leads to higher insider prices if the level of efficiencies is on one side of $e_{p}$ and lower insider prices on the other side of $e_{p}$. To be more specific, if $\partial p_{I}^{M} / \partial e<0$ so that the insider prices decrease with merger efficiencies for $e$ over the entire interval [0,1], we have the familiar situation where efficiencies mitigate the upward pricing pressure caused by the merger. In that case, the insiders' post-merger prices will fall below their pre-merger level if the size of merger efficiencies exceeds $e_{p}$. If $\partial p_{I}^{M} / \partial e>0$ at $e=e_{p}$, on the other hand, we have the intriguing situation where the merger leads to lower prices when efficiencies are small but higher prices when efficiencies are large.

\footnotetext{
${ }^{13}$ There is a literature, pioneered by Eckbo (1983), that uses the abnormal stock returns of outsiders to ascertain whether a merger is motivated by efficiency gains. Schumann (1993) criticizes this approach by arguing that "practically any pattern of rivals' abnormal returns can be consistent with some story of predominately procompetitive or anticompetitive mergers." Proposition 5 lends theoretical support to Schumann's argument because it implies that merger efficiencies can cause the abnormal stock returns of outsiders to go in either direction.
} 
Proposition 6. If condition (12) holds at $e=e_{p}$, there exists an $e_{l} \in\left(e_{\pi}, e_{p}\right)$ such that $p_{I}^{M}<p_{I}^{C}$ for $e \in\left(e_{l}, e_{p}\right)$, and an $e_{h} \in\left(e_{p}, 1\right)$ such that $p_{I}^{M}>p_{I}^{C}$ for $e \in\left(e_{p}, e_{h}\right)$.

Proof. Recall that $p_{I}^{M}=p_{I}^{C}$ and $p_{O}^{M}=p_{O}^{C}$ at $e=e_{p}$. By Proposition 1, condition (12) implies that $\partial p_{I}^{M} / \partial e>0$ at $e=e_{p}$. Then the result follows from the continuity of $p_{I}^{M}$ in $e$.

It is interesting to consider the implications of Proposition 6 for merger enforcement. In the literature, numerous retrospective studies have been conducted to assess the actual effects of past merger cases that were reviewed but not challenged by antitrust authorities in the US or elsewhere. ${ }^{14}$ Among these cases, at least two mergers are found to have generated efficiencies yet have led to higher prices. ${ }^{15}$ The conventional interpretation for such a finding is that the merger efficiencies were not large enough to offset the effects of increased market power conferred by the merger. Proposition 6, on the other hand, suggests another possible explanation. That is, instead of being a mitigating factor, merger efficiencies may actually have contributed to the price increases.

Therefore, Proposition 6 suggests that in cases where firms engage in quantity competition, antitrust authorities should not presume that merger efficiencies will necessarily counteract the anticompetitive effects of the merger. The merger can lead to higher prices because of, rather than despite of, large efficiencies.

\footnotetext{
${ }^{14}$ See Hunter et al. (2008) and Ashenfelter et al. (2014) for surveys of these studies.

${ }^{15}$ In the first case, SCM Corp., a producer of titanium dioxide $\left(\mathrm{TiO}_{2}\right)$, acquired a manufacturing facility of its competitor, Gulf \&Western, in 1983. After the merger, SCM replaced the old high-cost manufacturing process that had been used at the Gulf \& Western plant since 1964 with a proprietary low cost process. Despite the significant cost reduction, an econometric analysis by Schumann et al. (1992) finds that the merger caused the price of $\mathrm{TiO}_{2}$ to rise by more than $30 \%$ in the five-year period after the merger. In the second case, two brewers, Miller and Coors, established a joint venture to combine their operations in 2008. According to an analysis by Ashenfelter et al. (2015), the merger increased market concentration and reduced shipping costs. On average, the higher price caused by increased concentration was offset by a nearly equal and opposite efficiency effect. However, estimates for individual markets show that the merger caused the prices to rise in 26 of the 48 markets (Ashenfelter et al., 2015 p344).
} 
Finally, we end this section by noting that $e_{p}$ is a critical value for the outsiders' prices as well. That is, the post-merger prices of the outsiders are higher than their pre-merger prices if the level of efficiencies is on one side of $e_{p}$ and lower than their pre-merger counterpart on the other side of $e_{p}$. To be more specific, if condition (21) in Proposition 3 is satisfied, the merger leads to lower prices for the outsiders if $e<e_{p}$ and higher prices if $e>e_{p}$. The opposite is true if (21) does not hold.

\section{Consumer Welfare}

In this section, we investigate the effects of merger efficiencies on consumer welfare.

Propositions 1 and 3 imply that larger merger efficiencies would cause the prices of both insiders and outsiders to rise if conditions (12) and (21) hold in the post-merger equilibrium. In that scenario, merger efficiencies unambiguously reduce consumer welfare.

However, as noted in section 3, condition (21) does not hold in the case of linear demand functions. This raises the question: can conditions (12) and (21) both be satisfied in a postmerger equilibrium?

To answer this question, we consider a utility function that generates a system of non-linear demand functions and demonstrate that, under the conditions specified below, merger efficiencies indeed lead to higher prices for all goods and hence reduce consumer welfare.

Specifically, suppose that the market demand functions are generated from the following utility function of a representative consumer:

$$
\begin{aligned}
u\left(\mathbf{q}_{I}, \mathbf{q}_{o}\right)+y & =\sum_{i=1}^{2} \alpha_{I} q_{i}-\frac{q_{1}^{2}}{2}-\frac{q_{2}^{2}}{2}-\gamma q_{1} q_{2}-\delta q_{1} \sum_{i=3}^{n} q_{i}-\delta q_{2} \sum_{i=3}^{n} q_{i} \\
& +\sum_{i=3}^{n} \alpha_{O} q_{i}+\frac{\beta}{\tau} \sum_{i=3}^{n} q_{i}^{\tau}-\frac{\delta}{2}\left[\sum_{i=3}^{n} q_{i} \sum_{j=3}^{n} q_{j}-\sum_{i=3}^{n} q_{i}^{2}\right]+y
\end{aligned}
$$


where $y$ denotes the quantity of a numeraire good, $\alpha_{I}$ and $\alpha_{O}$ are positive parameters, and each of $(\gamma, \delta, \tau)$ is strictly between 0 and 1 . It is easy to verify that $u\left(\mathbf{q}_{I}, \mathbf{q}_{o}\right)$ displays diminishing marginal utility. As shown in Appendix B, moreover, $u\left(\mathbf{q}_{I}, \mathbf{q}_{o}\right)$ is concave in $\left(\mathbf{q}_{I}, \mathbf{q}_{o}\right)$ for quantities in the relevant range.

Solving the utility-maximization problem associated with (26), we obtain the following system of inverse demand functions:

$$
\begin{gathered}
p_{1}=\alpha_{I}-q_{1}-\gamma q_{2}-\delta \sum_{j=3}^{n} q_{j} \\
p_{2}=\alpha_{I}-\gamma q_{1}-q_{2}-\delta \sum_{j=3}^{n} q_{j} \\
p_{i}=\alpha_{o}+\beta q_{i}^{\tau-1}-\delta \sum_{j \neq i} q_{j}(i=3,4, \ldots, n)
\end{gathered}
$$

The main difference between this demand system and the linear demand system (14)-(16) is that the demand function for each outsider is non-linear in its own quantity. But the demand function for an insider remains linear, with $\gamma$ measuring the degree of substitutability between the insiders and $\delta$ indicating the degree of substitutability between an insider and an outsider.

In a symmetric equilibrium, (26) can be written in the form:

$$
\begin{aligned}
V= & 2 \alpha_{I} q_{I}-(1+\gamma) q_{I}^{2}-2(n-2) \delta q_{I} q_{o}+\alpha_{o}(n-2) q_{o}+\frac{\beta}{\tau}(n-2) q_{o}^{\tau} \\
& -\frac{\delta}{2}(n-2)(n-3) q_{o}^{2}-2 p_{I} q_{I}-(n-2) p_{O} q_{o} .
\end{aligned}
$$

We use (30) to measure consumer welfare before and after the merger.

Proposition 7. Suppose the market demand functions are represented by (27)-(29). A larger $e$ leads to higher prices for the insiders if and only if

$$
\gamma<\frac{2(n-2) \delta^{2}}{(n-3) \delta+\tau(1-\tau) \beta\left(q_{O}^{M}\right)^{\tau-2}}-1,
$$


holds in the post-merger equilibrium. A larger $e$ unambiguously raises the prices of the outsiders. Consequently, a larger $e$ reduces consumer welfare if (31) is satisfied.

Proof. Noting that $\Psi_{I}^{\prime}=-1, \Phi_{I}^{\prime}=-\gamma, \Psi_{O}^{\prime}=-(1-\tau) \beta\left(q_{O}^{M}\right)^{\tau-2}, \Phi_{O}^{\prime}=-\delta$ and $\Psi_{O}^{\prime \prime}=(2-$ $\tau)(1-\tau) \beta\left(q_{O}^{M}\right)^{\tau-3}$, we rewrite (12) to find (31). Condition (21) is satisfied because $\Psi_{O}^{\prime}+$ $q_{O}^{M} \Psi^{\prime \prime}{ }_{O}=(1-\tau)^{2} \beta\left(q_{O}^{M}\right)^{\tau-2}>0$. Proposition 3 then implies that a larger $e$ raises the prices of the outsiders. Consumer welfare falls with a larger $e$ because the indirect utility function associated with (26) is a decreasing function in prices $p_{I}$ and $p_{O}$.

Therefore, larger merger efficiencies will harm consumers if condition (31) is satisfied. As shown in Appendix B, a necessary condition for (31) to hold is that

$$
\delta>(1+\gamma) \frac{(n-3)}{2(n-2)} .
$$

This is consistent with our earlier observation that merger efficiencies lead to higher prices for insiders if the substitutability between an insider and an outsider is high while that between the insiders is low.

One drawback of (31) is that it contains an endogenous variable, $q_{O}^{M}$, which itself depends on the values of parameters such as $\gamma$ and $\delta$. To demonstrate clearly that there indeed exist parameter values for which the condition in Proposition 7 is met, we use a set of numerical examples.

Specifically, we present here numerical examples for different values of $e$ with the values of other parameters fixed at $n=12, \beta=0.14, \gamma=0.3, \delta=0.8, \tau=0.2, \alpha_{I}=70, \alpha_{O}=44$, and $c=1.8$. Additional technical details about the numerical simulations can be found in Appendix B. Below we display two diagrams to demonstrate our main results. 


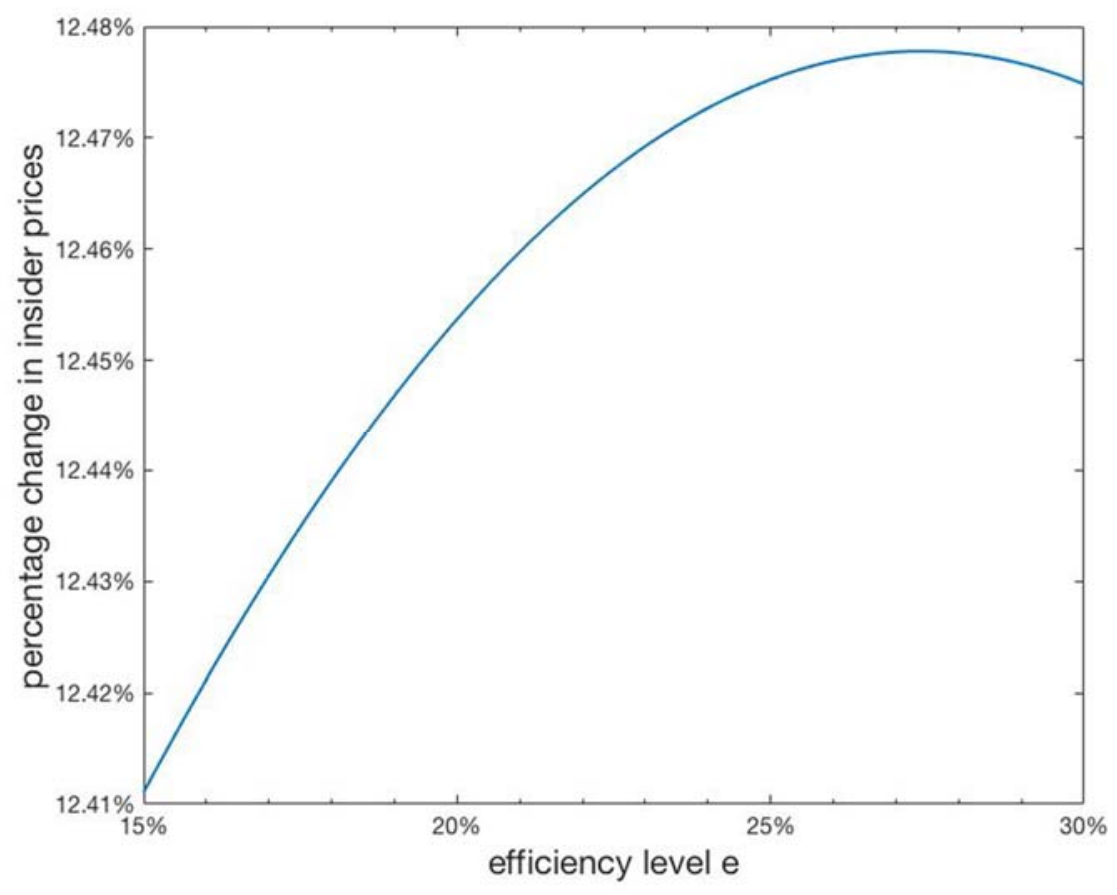

Figure 1: Impact of Merger on Insider Prices

Figure 1 illustrates the post-merger increase in the insider prices for $e \in[0.15,0.30]$. We choose this range of $e$ because, on the one hand, the merger is not profitable for $e<0.15$, and, on the other hand, condition (31) is violated and hence the insider prices fall with merger efficiencies for $e>0.27$. On the vertical axis of Figure 1 is the change in the prices of the insiders, $\left(p_{I}^{M}-p_{I}^{C}\right) / p_{I}^{C}$. From Figure 1 we see that, for $e$ in the stated range, the merger causes the insider prices to rise by more than $12 \%$, and larger efficiencies push the prices to move even higher.

Figure 2 demonstrates the impact of merger efficiencies on consumer welfare. On the vertical axis is the change in consumer welfare, $\left(V^{M}-V^{C}\right) / V^{C}$, where $V^{C}$ and $V^{M}$ denote the value of (30) in pre-merger and post-merger equilibrium, respectively. Figure 2 shows that for $e \in[0.15,0.30]$ the merger reduces consumer welfare by more than $19 \%$, and larger efficiencies exacerbate the reduction in consumer welfare. 


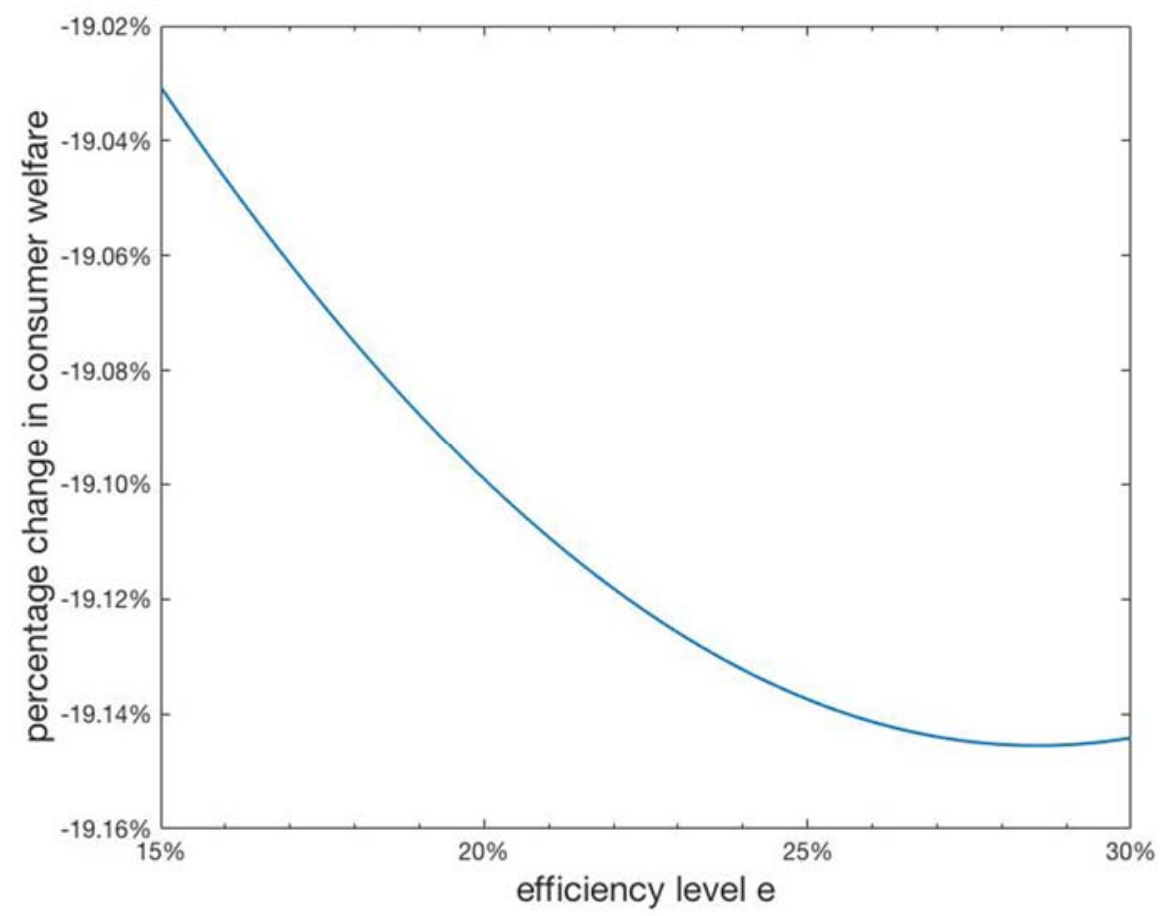

Figure 2: Impact of Merger on Consumer Welfare

Note that among the parameter values used in the numerical examples, $\gamma=0.3$ and $\delta=0.8$. That is, the degree of substitutability between the insiders is substantially smaller than that between an insider and an outsider. Therefore, these examples represent a situation where the products offered by an insider and an outsider are very close substitutes while those offered by the two insiders are distant substitutes. Moreover, the merger generates significant efficiencies in excess of $15 \%$. Yet the merger leads to substantial increase in prices and causes significant harm to consumers.

\section{Conclusions}

To answer the question posed in the title of this paper, merger efficiencies do not necessarily mitigate the price increases arising from the loss of competition. Our analysis demonstrates that when firms compete in quantity, lower marginal costs after a merger can exert upward rather than downward pressure on the prices of the merging firms. A policy implication of our results is that 
in cases where firms engage in quantity competition, a competition authority can no longer presume that merger efficiencies will necessarily offset the anticompetitive effects of the merger. The merger can lead to higher prices because of, rather than despite of, large efficiencies.

Finally, it is important to recognize that our results are obtained under a particular set of assumptions in a particular theoretical framework. Among the assumptions is that firms compete in quantity. If, instead, firms choose prices, the economic force that causes the insiders' prices to rise with merger efficiencies may not be present. While a formal analysis is beyond the scope of the present paper, it is our belief that merger efficiencies will not lead to higher prices when firms compete in prices. This is because prices are, in a standard model of oligopoly, strategic complements. Strategic complementarity implies that when the insiders reduce their prices in response to lower marginal costs, the outsiders will follow suit. This, in turn, will reinforce the insiders' incentives to cut prices. Consequently, it appears unlikely that larger merger efficiencies would lead to higher prices when firms compete in price. Therefore, a careful consideration of the nature of strategic interactions among firms is needed when applying our theory to a merger analysis. 


\section{References}

Amir, R., E. Diamantoudi and L. Xue, 2009, Merger performance under uncertain efficiency gains, International Journal of Industrial Organization 27, 264-273.

Ashenfelter, O., D. Hosken and M. Weinberg, 2014, Did Robert Bork understate the competitive impact of mergers? Evidence from consummated mergers, Journal of Law and Economics 57, S67-S100.

Ashenfelter, O., D. Hosken and M. Weinberg, 2015, Efficiencies brewed: Pricing and consolidation in the US beer industry, RAND Journal of Economics 46, 328-361.

Banal-Estañol, A., I. Macho-Stadler and J. Seldeslachts, 2008, Endogenous mergers and endogenous efficiency gains: The efficiency defense revisited, International Journal of Industrial Organization 26, 69-91.

Cheung, F.K., 1992, Two remarks on the equilibrium analysis of horizontal merger, Economics Letters 40, 119-123.

Eckbo, E.B., 1983, Horizontal mergers, collusion, and stockholder wealth, Journal of Financial Economics 11, 241-273.

Farrell, J. and C. Shapiro, 1990, Horizontal mergers: An equilibrium analysis, American Economic Review 80, 107-126.

Fisher, A., F. Johnson and R. Lande, 1989, Price effects of horizontal mergers, California Law Review 77, 777-827.

Froeb, L.M. and G.J. Werden, 1998, A robust test for consumer welfare enhancing mergers among sellers of a homogeneous product, Economics Letters 58, 367-69. 
Geiger, F. and D. Schiereck, 2014, The influence of industry concentration on merger motives empirical evidence from machinery industry mergers, Journal of Economics and Finance $38,27-52$.

Hunter, G., G.K. Leonard and G.S. Olley, 2008, Merger retrospective studies: A review, Antitrust Magazine 23, 34-41.

Jovanovic, D. and C. Wey, 2012, An equilibrium analysis of efficiency gains from mergers, DICE Discussion Paper, No. 64.

Levin, D., 1990, Horizontal mergers: The 50-percent bench-mark, American Economic Review $80,1238-1245$.

Lichtenberg, F., and D. Siegel, 1992, Productivity and changes in ownership of manufacturing plants, in Corporate Takeovers and Productivity, F. Lichtenberg, ed., Cambridge, Massachusetts: The MIT Press, 25-43.

Lommerud, K. E. and L. Sørgard, 1997, Merger and product range rivalry, International Journal of Industrial Organization 16, 21-42.

Motta, M. and H. Vasconcelos, 2005, Efficiency gains and myopic antitrust authority in a dynamic merger game, International Journal of Industrial Organization 23, 777-801.

McGuckin, R. H. and S. V. Nguyen, 1995, On productivity and plant ownership change: New evidence from the LRD, RAND Journal of Economics 26, 257-276.

Ollinger, M. and S.V. Nguyen, 2003, Empirical evidence on the motives for mergers and acquisitions in eight food industries, paper prepared for presentation at the American Agricultural Economics Association Annual Meeting, Montreal Canada, July 27-30, 2003. 
Salant, S.W., S. Switzer, and R.J. Reynolds, 1983, Losses from horizontal merger: The effects of an exogenous change in industry structure on Cournot-Nash equilibrium, Quarterly Journal of Economics 98, 185-199.

Salop, S., 1987, Symposium on mergers and antitrust, Journal of Economic Perspectives 1, 3-12.

Schumann, L., J. D. Reitzes, and R.P. Rogers, 1992, Case studies of the price effects of horizontal mergers, Bureau of Economics, Federal Trade Commission, retrieved from https://www.ftc.gov/sites/default/files/documents/reports/case-studies-price-effectshorizontal-mergers/232213.pdf, on November 25, 2016.

Schumann, L., 1993, Patterns of abnormal returns and the competitive effects of horizontal mergers, Review of Industrial Organization 8, 679-696.

Werden, G., 1996, A robust test for consumer welfare enhancing mergers among sellers of differentiated products, Journal of Industrial Economics 44, 409-413. 


\section{Appendix A. Linear Demand System}

In this appendix, we present technical details associated with the special case of linear demand functions. To simplify presentation, we define $A \equiv(n-3) \delta+2$ and $B \equiv(n-2) \delta$. Since $\delta<$ 1 , it can be verified that $A>B$.

It is clear that the linear demand functions (14)-(16) satisfy Assumptions 1 and 2. Regarding Assumption 3, it is easy to verify that

$$
J=2 \gamma[2+(n-3) \delta]+2\left(2-\delta^{2}\right)+2(n-3) \delta(1-\delta)>0 .
$$

Applying (5) to the linear demand functions, we derive the pre-merger equilibrium quantity of each insider and each outsider:

$$
q_{I}^{C}=\frac{(\alpha-c)(2-\delta)}{(2+\gamma) A-2 \delta B}, \quad q_{O}^{C}=\frac{(\alpha-c)(2+\gamma-2 \delta)}{(2+\gamma) A-2 \delta B}
$$

Using (A2) and the demand functions (14)-(16), we obtain the equilibrium prices before the merger:

$$
p_{I}^{C}=\frac{(\alpha-c)(2-\delta)}{(2+\gamma) A-2 \delta B}+c, p_{O}^{C}=\frac{(\alpha-c)(2+\gamma-2 \delta)}{(2+\gamma) A-2 \delta B}+c
$$

Solving (8)-(9) for the case of linear demand functions, we find post-merger equilibrium quantities and prices:

$$
\begin{gathered}
q_{I}^{M}=\frac{(\alpha-c)(2-\delta)+e c A}{2[(1+\gamma) A-\delta B]}, \quad q_{O}^{M}=\frac{(\alpha-c)(1+\gamma-\delta)-\delta e c}{(1+\gamma) A-\delta B}, \\
p_{I}^{M}=\frac{(\alpha-c)(1+\gamma)(2-\delta)-e c[(1+\gamma) A-2 \delta B]}{2[(1+\gamma) A-\delta B]}+c, \quad p_{O}^{M}=\frac{(\alpha-c)(1+\gamma-\delta)-\delta e c}{(1+\gamma) A-\delta B}+c .
\end{gathered}
$$

It is easy to verify that $\partial q_{I}^{M} / \partial e>0, \partial q_{O}^{M} / \partial e<0$, and $\partial p_{O}^{M} / \partial e<0$. Moreover, $\partial p_{I}^{M} / \partial e>0$ if and only if $(1+\gamma) A<2 \delta B$, i.e., if and only if (17) holds.

Regarding Assumption 4, we use (A2) and (A4) to find that $q_{I}^{M}>q_{I}^{C}$ at $e=1$ if and only if

$$
c>\frac{(2-\delta) \gamma \alpha}{(2-\delta) \gamma+(2+\gamma) A-2 \delta B}
$$


Note that the right-hand side of (A6) is less than $\alpha$. Thus, (A6) does not contradict $\alpha>c$.

\section{Appendix B. Nonlinear Demand System}

In this appendix, we present technical details associated with the special case of the non-linear demand system derived from the utility function (26). First, we present a condition that ensures the concavity of $u\left(\mathbf{q}_{I}, \mathbf{q}_{o}\right)$. It is easy to find

$$
\begin{gathered}
\frac{\partial^{2} u}{\partial q_{i}^{2}}=-1<0, \quad \frac{\partial^{2} u}{\partial q_{i} \partial q_{j}}=-\gamma, \quad \frac{\partial^{2} u}{\partial q_{i} \partial q_{k}}=-\delta, \quad(i, j=1,2, k=3, \ldots, n),(A 7) \\
\frac{\partial^{2} u}{\partial q_{i}^{2}}=-(1-\tau) \beta q_{i}^{\tau-2}<0, \quad \frac{\partial^{2} u}{\partial q_{i} \partial q_{j}}=-\delta, \quad(i, j=3, \ldots, n) .
\end{gathered}
$$

From (A7)-(A8), we see that $\partial^{2} u / \partial q_{i}^{2}<0$ for all $i=1,2, \ldots n$. To obtain the condition that ensures the concavity of $u\left(\mathbf{q}_{I}, \mathbf{q}_{o}\right)$, we calculate the pivots of the Hessian matrix associated with the utility function (26) without row exchanges or scalar multiplications of rows. To simplify the calculations, we evaluate the derivative $\partial^{2} u / \partial q_{i}^{2}$ at a symmetric equilibrium, i.e., at $q_{i}=q_{O}$ for $i=3, \ldots, n$. After straightfoward but tedious calculations, we can show that all pivots of the Hessian matrix are negative if each outsider's quantity satisfies

$$
q_{o}<\left[\frac{(1+\gamma)(1-\tau) \beta}{2(n-2) \delta^{2}-(1+\gamma)(n-3) \delta}\right]^{1 /(2-\tau)} .
$$

The parameter values used in the numerical simulations are chosen to ensure that they satisfy (A9) as well as Assumptions 1-3.

To derive condition (32), observe in (31) that

$$
\frac{2(n-2) \delta^{2}}{(n-3) \delta+\tau(1-\tau) \beta\left(q_{O}^{M}\right)^{\tau-2}}<\frac{2(n-2) \delta}{(n-3)} .
$$

Then (31) and (A10) imply 


$$
\gamma<\frac{2(n-2) \delta}{(n-3)}-1
$$

We rearrange (A11) to obtain (32). Note that the right-hand side of (32) is less than 1 because $1+\gamma<2$ and $n-3<n-2$.

The equilibrium quantities before the merger are determined by

$$
\begin{aligned}
& \alpha_{I}-(2+\gamma) q_{I}-(n-2) \delta q_{o}=c \\
& \alpha_{o}+\tau \beta q_{o}^{\tau-1}-(n-3) \delta q_{0}-2 \delta q_{I}=c
\end{aligned}
$$

The equilibrium quantities after the merger are solved from the equation system consisting of

$$
\alpha_{I}-(2+2 \gamma) q_{I}-(n-2) \delta q_{o}=(1-e) c
$$

and (A13).

The numerical simulations are conducted using the following two-step procedure. First, we choose a set of parameter values and solve (A12)-(A14) to find the equilibrium quantities. Then we use these quantities to verify whether Assumptions 1-3 and conditions (A9) and (31) are satisfied. The examples presented in section 6 are chosen from the sets of parameters that meet all of these restrictions. 